CHAPTER 4.9

\title{
ABERRATION-CORRECTED ELECTRON MICROSCOPY
}

THOMAS VOGT

4 Microscopy allows us to observe objects we cannot see with our eyes alone. With a light microscope, we can distinguish objects at 6 the scale of the wavelengths of visible light just under a micrometer. Around 1870 Ernst Abbe, who laid the foundation of modern s optics, suggested that the resolution of a microscope would improve by using some yet-unknown radiation with shorter wavelengths than visible light, that is, below 390 nanometers $\left(1 \mathrm{~nm}=10^{-9} \mathrm{~m}\right)$. Electrons can have wavelengths near 1 picometer $\left(1 \mathrm{pm}=10^{-12} \mathrm{~m}\right)$ and 2 should therefore allow atoms to be distinguished, since they are typically at least a few hundred pm apart. In this oversimplified view, 4 further decreasing the wavelength of the radiation should allow us to increase the resolution of an electron microscopy even more. However, this can only be done by increasing the energy of the radiation, which would ultimately destroy the samples. Other factors also affect the resolution of electron microscopes, among them non-ideal imaging properties of electromagnetic lenses, which result in false images. It took more than half a century to understand and control these aberrations and separate objects less than 1 Angstrom $\left(1 \AA=10^{-10} \mathrm{~m}\right)$ apart.

At the Technische Universität Berlin in 1931, Max Knoll and his doctoral student Ernst Ruska showed that magnetic coils could be used as lenses for electrons and thereby cleared the path to developing 
transmission electron microscopy (TEM) ${ }^{19}$ In TEM, a broad electron beam impinges on a sample and the electrons exiting the sample are magnified using an electro-optical lens. These "transmitted" electrons carry information about the structure of the material in the sample. A year after their first publication, the resolution limit of the light microscope was surpassed. The wavelength of electrons can be controlled by their accelerating voltage. It is about $4 \mathrm{pm}$ for a $100 \mathrm{keV}$ electron, smaller than an atomic diameter. In comparison, green light has a wavelength of about $550 \mathrm{~nm}$ and good light microscopes can separate two objects about $300 \mathrm{~nm}$ apart, which corresponds to about 1,000 atomic diameters.

Subsequently, Max Knoll and Manfred von Ardenne, who headed his private research laboratory, the Forschungslaboratorium für Elektronenphysik in Berlin-Lichterfelde made early attempts to scan a specimen with a fine electron beam and thereby also create an image. ${ }^{18,33}$ Although conventional TEM continued to dominate electron microscopy for over fifty years; the scanning technique was further developed in the 1960s by Albert Crewe and his collaborators in Chicago, ${ }^{9}$ leading to the imaging of heavy atoms in Scanning Transmission Electron Microscopy (STEM). ${ }^{8}$ This led to a revolution in sub-Ångstrom imaging in the late 1990s, when correcting aberrations associated with electro-optical lenses (as outlined in the next section) finally became possible. Today, STEM imaging is ubiquitous and routinely used in materials science and engineering as well as in condensed matter physics and chemistry. Many of the entries in this volume examine tools that were introduced to the materials research community, rapidly gained widespread acceptance, and then continued to slowly evolve. Other tools, however, struggled to live up to their promises, sometimes over many decades, before finally achieving success. STEM is a particularly clear instance of the latter category.

\section{Theoretical Reasons for Doubt and Hope}

In 1926 Hans Busch from Jena University described the focusing of electrons in a manner similar to how glass lenses can focus visible 
light. No glass or electromagnetic lens is perfect, but in light optics aberrations can be corrected by a series of convex and concave lenses. In electron optics this is not possible because there are no concave lenses. Thus, new ways of correcting for the aberrations caused by round electron lenses had to be developed.

The resolution of a STEM depends on the size of the electron beam one scans across the sample. In turn, the size of the beam depends on three main electro-optical effects. The first is the diffraction limit, which relates the resolving power to distinguish two objects and is proportional to the wavelength and inversely proportional to the illumination angle. Maximizing resolution would thus aim for high energies and small wavelengths of electrons as mentioned above and large illumination angles. The second is the spherical aberration of lenses: in STEM imaging, the smallest achievable electron probe is proportional to a measure of the deviation from perfect lensing called the constant of spherical aberration and the third power of the illumination angle. In a simple ray-tracing picture, this can be understood as creating a disk of least confusion before the image plane (see Figure 4.9.1). One should attempt to minimize both the wavelength and spherical aberration using appropriate optics. In contrast to the diffraction limit, one would aim for the smallest possible illumination angle. Third, the chromatic aberration in the image plane is due to the presence of electrons with different energies coming from their source and creating a disk of confusion whose size is proportional to the chromatic aberration constant and the width of the energy distribution. At small illumination angles, the diffraction limit determines the resolution, while at larger ones the spherical aberration dominates, provided that the spherical and chromatic aberration constants are of similar orders of magnitude and the energy spread of the electrons is about $1 \mathrm{eV}$. STEM imaging using energies above $100 \mathrm{kV}$ will have spherical and not chromatic aberration as the main limiting effect.

Early work by Otto Scherzer at the Technische Hochschule Darmstadt pointed to the unavoidable fact that round electron lenses have positive spherical and chromatic aberration effects, which will limit the resolution of an electron microscope ("Scherzer limit") to about 
(a)

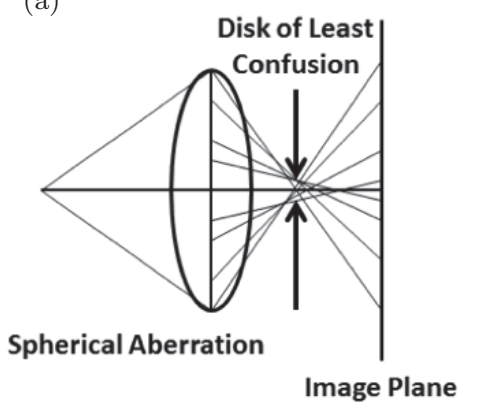

(b)
Disk of Confusion

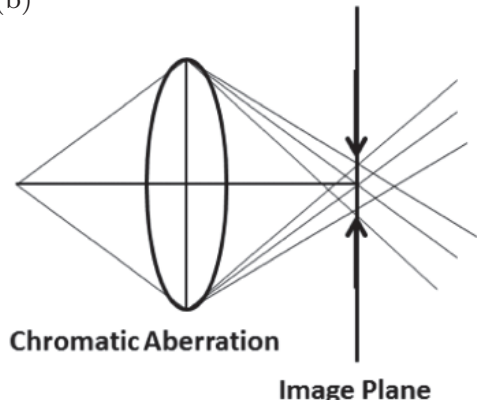

(c)

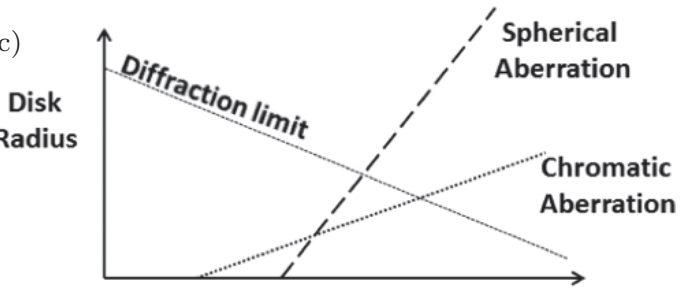

Illumination angle

Fig. 4.9.1. Ray-tracing of spherical (a) and chromatic (b) aberration effect in cylindrical electro-optical lenses and their dependence on the illumination angle for acceleration voltages higher than $100 \mathrm{kV}$. An electrostatic hexapole sketched to the right of the ray tracing of the spherical aberration effect allows for its correction as it displaces the electrons with electric fields as indicated. Part (c) shows how diffraction limit, spherical aberration, and chromatic aberration vary with the radius of the disk of least confusion and the angle of the illuminating electron beam.

one hundred times the electron wavelength, which in a TEM is near 2 2-2.5 picometers. ${ }^{30}$ In electron optics, round lenses will always dis3 play a positive aberration and no negative aberration is possible.

4 But in a groundbreaking paper in 1947, Scherzer showed that both 5 chromatic and spherical axial aberrations could be corrected by devi6 ating from round lenses with a rotationally symmetric electromag7 netic field using an electromagnetic multipole. ${ }^{29}$ He predicted, based 8 on a "gut-feeling" (German: rein gefuehlsmaessig) that this resulted 9 in a negative correction effect to counteract the positive aberration. ${ }_{10}$ Think of a multipole as a geometrical figure approximating a sphere ${ }_{11}$ by placing points on the surface of the sphere and connecting them. ${ }_{12}$ Rotating a quadrupole, hexapole, or octupole, made of electrical 
fields pointing in different directions, only approximates the rotational symmetry of the sphere and it is this small deviation from the sphere's shape that needed for aberration correction. A sketch is given in Figure 4.9.1]

\section{The First Arduous Steps toward Aberration Correction}

Between 1949 and 1954, Robert Seeliger, a student of Scherzer, was the first who attempted to build a corrector for a TEM comprised of two round lenses and three octupoles; however, he failed to overcome mechanical and electromagnetic instabilities. ${ }^{31}$ Nevertheless, he proved Scherzer's conjecture that such a multipole corrector leads to a negative spherical aberration needed to compensate for the positive one of the round objective lens. Seeliger's corrector was subsequently moved to the University of Tübingen where Gottfried Möllenstedt intentionally enhanced the spherical aberration by using an unusually large illumination angle to degrade the resolution (see Figure 4.9.1). ${ }^{24}$ Using octupoles, he then improved the resolution by about a factor of seven and significantly increased the contrast. Largely ignored work by Möllenstedt's PhD student Werner E. Meyer at the University of Tübingen showed that misalignment, static imperfections, and charging, and alternating external electromagnetic fields, as well as mechanical instabilities, were the main roadblocks for aberration correction. ${ }^{23}$ This proved to be correct until the 1990s! Geoffrey Archard, ${ }^{1}$ working at the Associated Electrical Industries (AEI) research laboratory at Aldermaston Court, built on this early work establishing that cylindrical lenses can be substituted by quadrupoles. He pointed out that an early attempt by Jack C. Burfoot at the University of Cambridge could be simplified by using a sequence of four optical elements: a quadrupole-octupole, a round lens-octupole, a second quadrupole-octupole, followed by a quadrupole. In 1964 Hans Deltrap built the first quadrupole-octupole corrector to eliminate spherical aberration. ${ }^{5,12}$ However, the resolution was limited not by the spherical resolution, but other qualities of the lenses at that time and his corrector was only tested on an electron-optical bench not in an electron microscope. 
An important international workshop took place in 1966 at Argonne National Laboratory, at which the design of a high-voltage, high-resolution electron microscope with a quadrupole-octupole corrector was proposed but subsequently not funded. In 1971, Harald Rose at TU Darmstadt, after showing that all correctors to date suffered from off-axis coma, a "comet-like blur away from the optical axis," developed a corrector that could simultaneously correct for chromatic and spherical aberration. ${ }^{27}$ In 1972 Crewe and Vernon - Beck made new attempts to correct for spherical aberration of a STEM using a quadrupole-octupole corrector alleviating some of the difficulties encountered in the Darmstadt project. ${ }^{7}$ However, they were unable to eliminate aberrations due to inhomogeneity of the metal used in the lens and the lack of fast diagnostic and control electronics to establish suitable optical settings for the many parameters. V@ such electronics, no feedback control was possible. At the International Congress on Electron Microscopy in Toronto in 1978, Scherzer quipped that the resolution of the best uncorrected TEMs "is clearly limited by the unavailability of the necessary funds." ${ }^{28}$ The Darmstadt project continued until Scherzer's death in 1982, when it was terminated despite significant progress. ${ }^{4}$

Up to this point, techniques using sextupole correctors had been ignored since Peter Hawkes had shown in 1965 that the large second-order aberrations prevent the exploitation of the favorable third-order aberrations to correct for spherical aberration of round lenses. ${ }^{16}$ In an important breakthrough, Beck showed in 1979 that one can arrange sextupoles in a way to suppress this large secondorder aberration. ${ }^{3}$ This design was subsequently improved by Rose and later morphed into a prototype of the sextupole correctors which are now produced commercially by the CEOS Company in Heidelberg. ${ }^{26}$

\section{The Darkest Hour... and a New Dawn}

In the late 1980s "aberration fatigue" set in after the Darmstadt project ended with Scherzer's death. Crewe gave up, saying "unfortunately, we could never make the corrector work.... After 
many heartbreaking attempts, we were forced to admit defeat," and the US National Science Foundation decided to no longer fund efforts to build correctors. ${ }^{15}$ The materials science community, which had always been seen as the main user of high-resolution imaging, set their hopes on high-voltage electron microscopy where resolution was simply enhanced by reducing the wavelength of the electron beam (see diffraction-limit above and Figure 4.9.1k) by going to acceleration voltages of 1 million volts and higher. These gigantic instruments reached Angstrom resolution and the reduced electron inelastic scattering at such high energies allowed the use of thicker samples. However, atom displacement damage ("knock-on" damage) destroyed the samples, often within minutes.

There is a certain irony that funding agencies in Europe, Japan, and the United States were prepared to invest an order of magnitude more in high-voltage instruments (one microscope would cost tens of millions of US dollars) than was ever allocated for aberration correction of electron lenses. During these dark days for the aberration-correction community, it was realized that the required machining, manufacturing, and positioning of the many required optical elements was beyond what could reproducibly be achieved at that time. Furthermore, neither the stable hardware (that is, power supplies) nor the software existed to excite the various multipoles and other optical elements and integrate them with diagnostic tools based on fast detector readout to ensure feedback for auto-tuning algorithms. As Harold Rose was quoted by Knut Urban, "it took more time to adjust a specific electron optical state than this would have time to last." " Also, a quadrupole-octupole corrector is not very suitable for conventional TEM as one needs to correct over the whole region imaged. A much more suitable imaging system would be to correct a probe-forming SEM or STEM. Fortunately, the electron optics community proved to be resilient and was ultimately able to complete the revolution in high-resolution electron imaging after a long march of over four decades. Three main efforts in Europe funded internally by the European Molecular Biology Laboratory (EMBL), privately by the Volkswagen Foundation and by the Paul Instrument Fund of the Royal Society in the 
United Kingdom provided the funds needed to realize imaging at the atomic scale.

Due to the intrinsic difficulty in applying quadrupole correctors to TEM, Max Haider convinced management that a SEM with spherical and chromatic corrections aligned with the mission of EMBL as it had the potential to be used for imaging larger biomolecules. EMBL had a tradition of instrument development and could support this effort by in-house funding. With Joachim Zach, Haider proved the working principle of a quadrupole-octupole corrector using a low-voltage SEM. Despite its intrinsic lower resolution compared to a TEM, the instrument's resolution was improved from 5.6 to $1.8 \mathrm{~nm} .{ }^{34,35}$ Their design was a corrector based on Hardy's thesis from 1967 in Cambridge.

Parallel to these efforts Haider, Rose, and Urban lobbied for and received high-risk funding from the Volkswagen Foundation which allowed them to design, build, and test a hexapole-based corrector at the EMBL in Heidelberg. Rose's corrector used a telescope round lens doublet and two identical sextupoles, one centered at the front focal point of the first round lens and the other on the back focal point of the second round lens. The hexapole arrangement cancels out all second order path deviations, whereas the third order rotationally symmetric path deviations add up. The result is a negative spherical aberration proportional to the square of the hexapole strength compensating the positive one of the objective lens. The prolonged struggle to secure funding is described in detail in Urban's 2015 paper. The final decision by the Volkswagen foundation was made by a one-vote majority and only part of the requested funding was granted; the rest was contingent on achieving a critical milestone showing that aberration correction could be achieved on a conventional TEM, a worst-case test as mentioned above. First images were obtained on a Philips CM200 microscope with a Rose corrector in Heidelberg by Bernd Kabius on June 24, 1997, before the instrument was shipped to Jülich since EMBL management had decided to shut down in-house electron microscopy development. He showed that one could resolve dumbbells (two atoms in close proximity in a projection) $1.4 \AA$ apart when viewing along the (110) direction in a GaAs 
sample. A paper was submitted first to Nature and then Science and initially rejected by both but finally accepted in Nature. ${ }^{14}$

During the same period, Ondrej Krivanek and Niklas Delby con4 tinued to work on adapting quadrupole-octupole correctors to a 5 Vacuum Generator (VG) STEM at the Cavendish Laboratory in - Cambridge. They were funded by the Royal Society (Paul Instrument Fund) and now had access to high speed diagnostic and control $\therefore$ electronics to tune the corrector. Furthermore, they installed addi9 tional corrector coils to cancel parasitic aberrations. These improve10 ments allowed them to present initial results in 1997 — one hundred 11 years after the discovery of the electron by J. J. Thomson. ${ }^{21}$ Impres${ }_{12}$ sive results using dark field (DF) imaging were published in $1998 .{ }^{20}$ 13 Subsequently Krivanek and Delby moved to Seattle and started the 14 company NION (NIklas and ONdrei). In 2000 their MARK-2 correc15 tor was working and installed in a VG STEM at a specially shielded 16 site at IBM where it allowed DF images at a resolution of $1.4 \AA$ to ${ }_{17}$ be recorded. An iconic image of silicon dumbbells (Figure 4.9.2) is shown in a 2001 paper. ${ }^{11}$ The same instrument at IBM was used to record sub-Angstrom resolution images. ${ }^{2}$

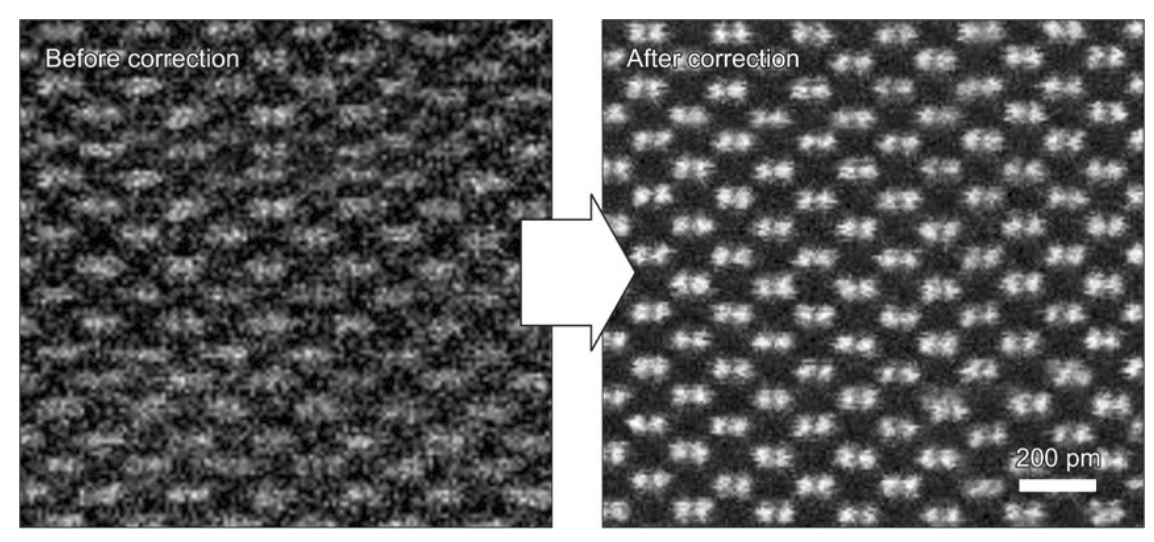

Fig. 4.9.2. Scanning TEM micrograph of silicon "dumbbells" before and after automatic aberration correction. Source: Inada H, et al. High-speed and sensitive $\mathrm{x}$-ray analysis system with automated aberration correction scanning transmission electron microscope. Applied Microscopy. 2015;45(1):1-8. Credit: Creative Commons CC BY-NC 3.0. 
As spherical aberration correction was being successfully implemented in commercially available electron microscopes, chromatic aberration was now the major hurdle to higher resolution. Many commercial electron microscopy manufacturers simply limited the energy spread of the incident beam by using a monochromator which minimized but did not correct for chromatic aberration.

After a workshop convened by Murray Gibson in July 2000, the TEAM (Transmission Electron Aberration-corrected Microscope) project was funded by the US Department of Energy involving the national laboratories at Argonne, Brookhaven, Oak Ridge, and Lawrence Berkeley as well as the Frederick Seitz Materials Research Laboratory at the University of Illinois. Harald Rose's new design replaced each sextupole in the original corrector by a telescopic quadrupole-octupole quintuplet. CEOS further optimized the design creating an achromatic aplanatic system with stabilities at the order of $2 \times 10^{-8}$ for all electric and magnetic quadrupole fields. FEI and CEOS built two instruments, TEAM 0.5, a TEM and STEM with spherical aberration correction, and TEAM 1, a STEM with spherical aberration correction and TEM with chromatic and spherical correction, which were installed at the National Center for Electron Microscopy at the Lawrence Berkeley National Laboratory in 2008. These best-in-class instruments have resolutions of 65 and $55 \mathrm{pm}$ at 200 and $300 \mathrm{kV},{ }^{17}$ respectively, and the STEM resolves a $47 \mathrm{pm}$ dumbbell spacing along the 114 direction in a germanium crystal. ${ }^{13}$

Samples containing light elements such as carbon, oxygen, or lithium degrade rapidly in an electron beam due to "knock-on" damage, where atoms are displaced from their original positions by high-speed electrons. However, reducing the accelerating voltage increases the damage due to radiolysis and chromatic aberration becomes dominant. At the University of Ulm in collaboration with two companies, FEI and CEOS, the SALVE (Sub-Ångstrom Low-Voltage Electron microscopy) project initiated by Ute Kaiser has shown spherical and chromatic aberration correction for accelerating voltages between 20 and $80 \mathrm{kV} .{ }^{22}$ Between 40 and $80 \mathrm{kV}$ sub-Ångstrom resolution was achieved. More recently, a Japanese collaboration by the microscopy manufacturer JEOL and the National 
1

Institute of Advanced Industrial Science and Technology built a TEM with a spatial resolution of $1.4 \AA$ at $15 \mathrm{kV} .{ }^{25}$ This allowed the observation of a monolayer of graphene at atomic resolution. This bodes well for the structural characterization of very beam-sensitive materials.

Over half a century of experimental struggles since the emergence of electron microscopes in 1932 allowed us to finally achieve the goal of sub-Ångstrom imaging. Although the key strategy, namely the deviation from round electro-optical elements by using multipoles, was recognized early on by Scherzer in 1947 and the first corrector was built by Robert Seeliger in the early 1950s, we had to wait until the 1990s until we could control the mechanical and electronic instabilities that were limiting the resolution. Only after these "secondary effects" were understood and controlled did aberration limit the resolution and could be corrected for. The length of such developments tests the patience of private and public funding agencies; however, transformative scientific advances are more and more becoming battles of attrition rather than singular strokes of geniuses.

\section{References}

1. Archard GD. An unconventional electron lens. Proceedings of the Physical Society. 1958;72(1):135-37.

2. Batson PE, Delby N, Krivanek OL. Sub-angstrom resolution using aberration-corrected electron optics. Nature. 2002;418:617-20.

3. Beck VD. A hexapole spherical aberration corrector. Optik. 1979;53:241-55.

4. Bernhard W. Erprobung eines sphaerisch korrigierten Elektronenmikroskops. Optik. 1980;57:73-94.

5. Burfoot J. Correction of electrostatic lenses by departing from rotational symmetry. Proceedings of the Physical Society. 1953;B66:775-92.

6. Crewe AV. Scanning electron microscopes: is high resolution possible? Science. 1966;154(3750):729-38.

7. Crewe AV. Some Chicago aberrations. Microscopy and Microanalysis. 2002;8(Suppl. 2):4-5.

8. Crewe AV, Wall J, Langmore J. Science. 1970;168:1338-40.

9. Crewe AV, Wall J, Welter LM. Journal of Applied Physics. 1968;39(13): 5861-68.

10. Dahmen U, Erni R, Kisielowki C, Radmilovic V, Ramasse Q, Schmid A, Duden T, Watanabe M, Minor A, Denes P. An update on the TEAM 
project - first results from the TEAM 0.5 microscope, and its future development. In Luysberg M, Tillmann K, Weirich T, editors. Proceedings of the $14^{\text {th }}$ European Microscopy Congress, Aachen, Germany, vol. 1. Berlin: Springer; 2008.

11. Delby N, Krivanek OL, Nellist PD, Batson PE, Lupini, AR. Progress in aberration-corrected scanning transmission electron microscopy. Journal of Electron Microscopy. 2001;50:177-85.

12. Deltrap JHM. Correction of spherical aberration with combined quadrupoleoctupole units. In Titlbach M, editor. Proceedings of the Third European Regional Conference on Electron Microscopy, Prague, vol. A, Prague: Czechoslovakian Academy of Sciences; 1964. 45-46.

13. Erni S, Rossell MD, Kiselowski C, Dahmen U. Atomic-resolution imaging with sub-50 nm electron probe. Physical Review Letters. 2009;102:096101.

14. Haider M, Uhlemann S, Schwan E, Rose H, Kabius B. Electron microscopy image enhanced. Nature. 1998;392:768-69.

15. Hawkes PW. The correction of electron lens aberrations. Ultramicroscopy. 2015;156:A1-A64.

16. Hawkes PW. The geometrical aberration of general optical systems. Philosophical Transactions of the Royal Society of London. 1965;A257:479-662.

17. Kisielowski C, Freitag B, Bischoff M, van Lin H, Lazor S, Knippels G, Tiemeijer P, van der Starn M, von Harrach S, Stekelenburg M, Haider M, Uhlemann S, Müller H, Hartel P, Kabius B, Miller D, Petrov I, Olson EA, Donchev T, Kenik EA, Lupini AR, Bentley J, Pennycook SJ, Anderson IM, Minor AM, Schmid AK, Duden T, Radmilovic V, Ramasse QM, Watanabe M, Erni R, Stach EA, Denes P, Dahmen U. Detection of single atoms and buried defects in three dimensions by aberration-corrected electron microscope with $0.5 \AA$ information limit. Microscopy Microanalysis. 2008;14:469-77.

18. Knoll M. Aufladepotentiel und Sekundäremission elektronenbestrahlter Körper. Zeitschrift für Technische Physik. 1935;16:467-75.

19. Knoll M, Ruska E. Das Elektronenmikroskop. Zeitschrift für Physik. 1932;78(5-6):318-39.

20. Krivanek OL, Delby N, Lupini AR. Towards sub- $\AA$ electron beams. Ultramicroscopy. 1999;78:1-11.

21. Krivanek OL, Delby N, Spence AJ, Camps RA, Brown LM. In Sturgess JM, editor. Proceedings of the Ninth International Congress on Electron Microscopy, Toronto, Canada, vol. 3. Toronto: Microscopical Society of Canada, 1978. 185-96.

22. Linck M, Hartel P, Uhlemann S, Kahl F, Müller H, Zach J, Haider M, Niestadt M, Bischoff M, Biskupek J, Lee Z, Lehnert T, Börrnert F, Rose H, Kaiser U. Chromatic aberration correction for atomic resolution TEM imaging from 20 to $80 \mathrm{kV}$. Physical Review Letters. 2016;117:076101.

23. Meyer W.E. Das praktische Aufloesungsvermoegen von Elektronenmikroskopen. Optik. 1961;18:101-14.

24. Moellenstedt G. Elektronenmikroskopische Bilder mit einem nach O. Scherzer sphaerisch korrigierten Objektiv. Optik. 1956;13:209-15. 
25. Morishita S, Mukai M, Suenaga K, Sawada H. Atomic resolution imaging at an ultralow accelerating voltage by a monochromatic transmission electron microscope. Physical Review Letters. 2016;117:153004.

26. Rose H. Correction of aperture, aberrations in magnetic systems with threefold symmetry. Nuclear Instruments and Methods in Physics Research. 1981;187:187-299.

27. Rose H. Elektronenoptische Aplanate. Optik. 1971;34:285-311.

28. Scherzer O. Limitations for the resolving power of electron microscopes. In Sturgess JM, editor. Proceedings of the Ninth International Conference on Electron Microscopy, vol. 3. Toronto: Microscopical Society of Canada;1978.

29. Scherzer O. Sphärische und chromatische Korrektur von Elektronenlinsen. Optik. 1947;2:114-32.

30. Scherzer O. Über einige Fehler von Elektronenlinsen. Zeitschrift für Physik. 1936;101:593-693.

31. Seeliger R. Spherical-aberration correction of electron lenses by means of image-forming elements without rotational symmetry. Optik. 1951;8:311-17.

32. Urban KM. In quest of perfection in electron optics: a biographical sketch of Harald Rose on the occasion of his $80^{\text {th }}$ birthday. Ultramicroscopy. 2015;151:2-10.

33. von Ardenne M. Das Elektronen-Rastermikroskop: Theoretische Grundlagen. Zeitschrift für Physik. 1938;109(9-10);553-72.

34. Zach J, Haider M. Aberration correction in a low voltage SEM by a multipole corrector. Nuclear Instruments and Methods in Physics Research Section A. 1995;363(1-2):316-25.

35. Zach J, Haider M. Correction of spherical aberration in a low voltage SEM. Optik. 1995;93:112-18. 


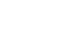

(n)

